\title{
Role of Technology in the Development of Smart Cities
}

\author{
Md. Shelim Miah ${ }^{1 *}$, Ruhul Amin ${ }^{2}$ \\ ${ }^{1}$ Assistant Professor, Department of Business Administration, Asian University of Bangladesh, Dhaka, BANGLADESH \\ ${ }^{2}$ Senior Data Entry Control Operator (IT), ED-Maintenance Office, Bangladesh Bank (Head Office), Dhaka, BANGLADESH \\ *Corresponding Contact: \\ Email: mmshelim@aub.edu.bd
}

\begin{abstract}
The key aim of this paper is to address the technical effects of smart city growth. While the smart city issue has been discussed in recent literature, it is of interest if macro ICT considerations should also be considered for determining a city's technical advancement. First of all, a literature analysis of a smart city is presented to accomplish this purpose. Along with a theoretical structure focused on the information community, an overview of the smart city idea is included. The ICT development of Smart Cities depends on the characteristics and features of the cities, as well as on macro-technological considerations. Cities that use information technology as a means of urban sustainability build smartness to emerge as smart cities as a source of constant growth and transformation within the urban ecosystem, pursuing a managerial and organizational vision of sustainability. The aim of this thesis is to suggest a theoretical overview of the city as a sustainable society that drives urban development and adopts a smart urban growth vision.
\end{abstract}

Key words:

Smart Cities, Technology, Smart City Challenges, Smart City Opportunities

\section{INTRODUCTION}

According to a 2014 report by the United Nations Population Fund, over sixty-six percent $(66 \%)$ of people will live in cities by 2030 . More people will move from rural areas to urban areas searching for a better life (Lea, 2017). As a result, resources will become overstretched in terms of security, environment and scalability as they try to support the rapidly increased population. There will be need to manage the billions of people and provide them with quality life (Chaudhari, 2017). In order to solve such problems, governments and engineers are leveraging technology to search for new approaches that will improve the management of resources in urban areas. The approaches will help perform various tasks, such as the improvement of transport, management of water and energy, waste management, including other resources and activities that underpin the operations of urban areas (Lea, 2017). Urban 
areas that rely on technological innovations to improve the efficiency of their operations and enhance the quality of life of the people are known as smart cities.

Smart Cities integrate various technologies that can deliver sustainable socio-economic development of urban areas. They rely on artificial intelligence, Internet of Things, Machine Learning, Deep Learning, and Big Data, among other technologies, to develop Smart Cities' applications that sustain and improve the lives of billions of people in urban areas (Donepudi, 2015). According to Voda \& Radu (2018), the use of technology in Smart Cities should be able to improve the quality of life of the people, stimulate growth in the economy and improve urban management.

The goal of this study is to explore the roles that Artificial Intelligence, Machine Learning, Deep Learning and Internet of Things can play in the development of smart cities. The paper provides a discussion of the applications of each of these technologies in the development of smart cities.

\section{Research Objectives}

Developing effective smart cities' applications requires a combination of multiple technologies. Therefore, understanding the role that emerging technologies can play in the development of smart cities can help develop efficient and effective applications that solve the socio-economic problems experienced in the highly-populated modern urban areas. This study explores the smart city concept and examines the role of emerging technologies, including Artificial Intelligence, Machine Learning, Deep Learning and Internet of Things, in the development of smart cities. The research will help strengthen the conceptual understanding of the role of emerging technologies in the development of smart cities.

\section{Research Methodology}

This research adopts a qualitative methodology. The methodology is based on the content analysis of scholarly and industry publications related to the development of smart cities. The research's main sources are journals, articles, and white papers related to the use of technology, especially artificial intelligence, machine learning, deep learning, and Internet of Things in smart cities' development.

\section{LITERATURE REVIEW}

\section{Smart City concept}

According to Arroub et al. (2016), urbanization processes are related to the socio-economic and environmental protection of urban areas. The population in cities around the world is rapidly increasing. Consequently, most of the cities around the world are at the height of seeking approaches that can solve the new challenges originating from population increment. For instance, they are seeking optimal solutions that can facilitate efficient utilization of resources, effective management of the environment, energy, water, safety, transport and public services (Arroub et al., 2016). This helps ensure that the billions of people living in urban areas and those expected in the future get a quality life. In this regard, the Smart City concept is slowly becoming a reality. In the $20^{\text {th }}$ century, Smart Cities were fictional works of science. But today, governments are initiating the development of smart cities, thanks to emerging technologies, telematics development and improved intelligence of devices. 
Furthermore, technology is essential to support the development of automated systems that enable individuals to monitor, understand and plan urban areas. Therefore, the smartness of cities relies on the creation of intelligent infrastructure and the connection between technology and people. Additionally, according to Arroub et al. (2016), the smartness of a city must respect various factors, including sustainability, smartness, and inclusiveness. Sustainability involves improving the city and environmental relationships and can include the use of a green economy. Smartness means governance and economy awareness, while inclusiveness involves fostering economic and social cohesion.

\section{Smart City Dimensions}

The Smart City development context has various dimensions. The dimensions include people, community and technology. A Smart City can only be smart when it highlights people, communities and technology, but not technology only (Pozdniakova, 2016).

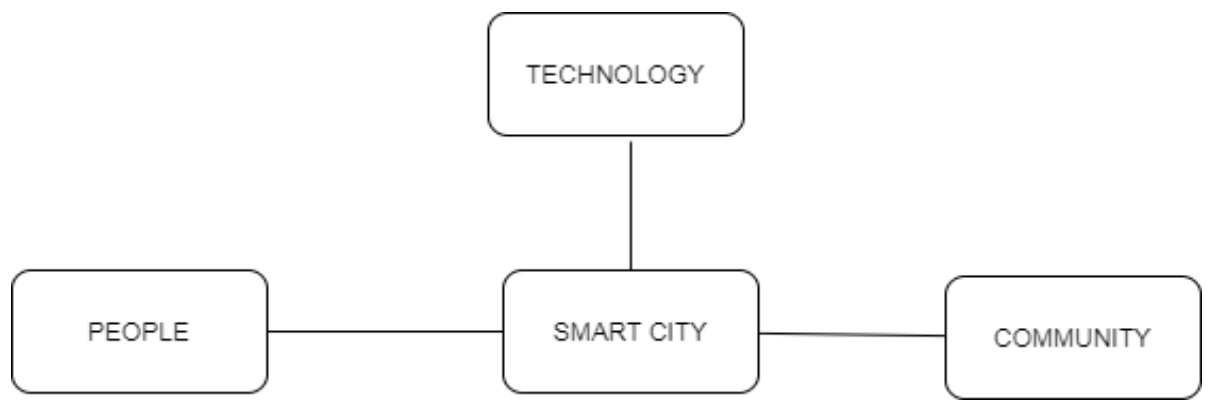

Fig 1: Smart City Dimensions

The presence of technology gives the Smart City an opportunity to have more creativity, improve governance and promote the quality of life. The people dimension segment includes a human city, a knowledgeable city, and human and social infrastructures necessary for the development of smart cities (Pozdniakova, 2016). In order to have a coherent smart city, the learning, cultural, and business aspects of smart people are necessary. They play an essential role in upgrading the competitively of urban areas contexts by helping develop an information economy workforce. Smart people are able to lead a life founded on better education and a skilled workforce (Arroub et al., 2016). Finally, smart communities aim at integrating the necessary number of city dwellers in Information Technology in order to improve their quality of life.

\section{SMART City Models}

In order to make smart cities real, various leading stakeholders, such as governments, businesses, and professionals, have been working on the development of effective smart city models. Although most developed cities around the world have made significant progress in the development of smart cities, small cities face numerous challenges as they work on implementing the new and disrupting smart city concept. According to Arroub et al. (2016), Smart City Models should be based on a comprehensive examination of current success stories on Smart City implementation. Additionally, Smart City implementation should consider the implementation of various smart city paradigms, which consist of aspects such as smart economy, smart mobility and transport, smart environment, smart living, smart governance, smart people and smart economy. 


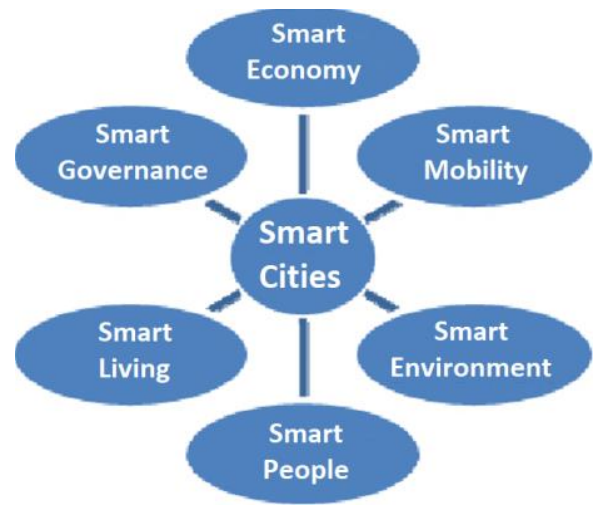

Fig 2: Smart City Aspects

Below is a brief discussion of the above smart city characteristics.

Smart Governance - Smart Cities involve multiple stakeholders handling different projects. To improve the management of Smart Cities' projects, the level of governance should be of high quality. Traditionally, the management of urban centers' projects was done manually. Today, Information Technology helps improve the management of Smart City projects through enhanced communication networks, improved communication systems, innovative policies and better business models (Mohanty et al., 2016; Arroub et al., 2016). Additionally, with Smart Governance, stakeholders can collaborate and cooperate on projects, have improved leadership models and solve issues efficiently.

Smart economy - The most important thing in a smart economy is the presence of smart companies that can produce innovative ideas on resource optimization and improvement of price-quality ratio (Arroub et al., 2016). The ideas should be able to improve productivity and reduce the cost of production. Additionally, smart companies should be able to achieve high levels of competitiveness through higher profits, good quality products and efficient costs of operation (Mohanty et al., 2016). Also, the smart economy should focus on sustainable use of energy resources and remain socially responsible.

Smart Environment - Due to the increased population in urban centers, cities must work on achieving a smart environment. Smart environments involve the use of green and natural energy resources with less pollution to the environment (Mohanty et al., 2016; Arroub et al., 2016). Waterways, sewers and green spaces should be managed in a smart manner.

Smart living - Smart Cities should support a smart living of the people. People should be able to develop intelligent ways to live through information technology. In Smart Cities, the interconnection of devices using information technology makes a lot of daily tasks carried out by the people easier, safer and cheaper (Mohanty et al., 2016). For example, a smart building may consist of numerous interconnected devices that allow managers to gather data, analyze the data and make appropriate decisions aimed at managing the building effectively. This allows a smart of living which smart cities should support.

Smart Mobility - Transport management is essential in all urban places. Cities experience various transport challenges, such as congestions and poor transport networks. Smart cities should be able to support various systems such as Transport Management Systems and Traffic Control systems, which have evolved over the years to support effective and smart mobility in cities (Arroub et al., 2016). In addition to such systems, smart cities can leverage 
technology to develop strategies that can meet the mobility requirements of current populations in urban centers and the expected growth of urban populations in the future.

Smart people - Urban centers cannot achieve smartness without the people being smart. The people must be educated, learned and knowledgeable (Arroub et al., 2016). In most cases, when talking about smart cities, the people dimension is forgotten. However, it is important to ensure that the smart people concept is implemented in order to achieve efficient cities where people can use the implemented smart technologies.

\section{Smart City Challenges}

Cities are exposed to various challenges, such as flooding and the greenhouse phenomenon. However, the main areas that, if not addressed, can limit the successful implementation of smart cities include economic, social, demographic, and environmental factors. These aspects must be highlighted when implementing smart cities. Furthermore, it is important to note areas such as transport and health, which are the main obstacles to achieving totally smart cities.

Transport congestion and pollution effects - Transport problems in cities because various challenges that affect the well-being of people. One main obstacle to achieving smart cities is the domination of private cars. The domination of private cars is a big problem for the future of smart cities, considering the large amount of energy that cars consume. Although smart cars provide owners with privacy and convenience, the level of air pollution they produce is a big problem (Arroub et al., 2016). They emit dangerous gases and noise that pollute the air and the environment in general. Additionally, the presence of many cars leads to increased congestion and accidents in cities. This brings a big challenge to the development of smart cities. It is problematic to develop transport systems that are able to fulfil the needs of the people in a green environment. Maybe a good measure could be the adoption of other methods of transport such as walking, cycling and use of public transport, which can reduce congestion and pollution and cities.

Healthcare obstacles - Healthcare is important in the improvement of the well-being of people. However, even people living in the most developed urban centers still face healthcare challenges such as overcrowded healthcare centers and high cost of healthcare. Despite the presence of technology-based solutions, many people still do not use them. In order to solve such challenges, stakeholders responsible for the implementation of Smart Cities should focus on electronic healthcare. Electronic healthcare can help reduce congestion and fasten the delivery of healthcare services to people with chronic infections. The various solutions that electronic healthcare provides to improve healthcare delivery include tele-care and tele-medicine, which help to provide healthcare services to patients regardless of their physical geographical locations. Medical care professionals can carry out disease diagnosis and recommend an appropriate medication to patients without the need to physically attend those (Arroub et al., 2016). Additionally, smart cities can adopt and implement $\mathrm{m}$-Health, which is essential in the improvement of communication, sensing and monitoring of healthcare data in order to provide real-time healthcare results to patients. As a result, the healthcare obstacle to the development of smart cities is solved. 


\section{Smart City Opportunities}

Smart cities provide numerous opportunities for city dwellers. Some of these opportunities include sustainable transportation systems and a green environment.

Sustainable transportation systems - One of those opportunities is sustainable transportation systems, which is achieved through the smartification of traffic systems. As discussed earlier, Smart Cities can rely on smart transport management systems that have the capability to reduce congestion on roads, provide citizens with an improved travel experience and reduce the time take to respond to incidents (Donepudi, 2015). A good transport management system should be able to support various features that include the ability to sense and gather data through road sensors, social media, and the use of cameras. The data collected can be analyzed in real-time, allowing the faster making of decisions related to transport systems.

Green Environment - Achieving a green environment is a goal that Smart Cities can achieve. There is increased awareness of the bad effects of environmental pollution and the need to use renewable energy such as solar power and wind power. Additionally, smart cities can rely on technologies such as Artificial Intelligence to make energy generation, consumption and management smarter. Efficient energy management can be achieved through the creation of smart grids that can route energy to consumers in a smart, effective and efficient manner.

The chart below shows the predicted smart city market in various years.

\section{Global smart city market (US\$ billion), $2018-2025$}

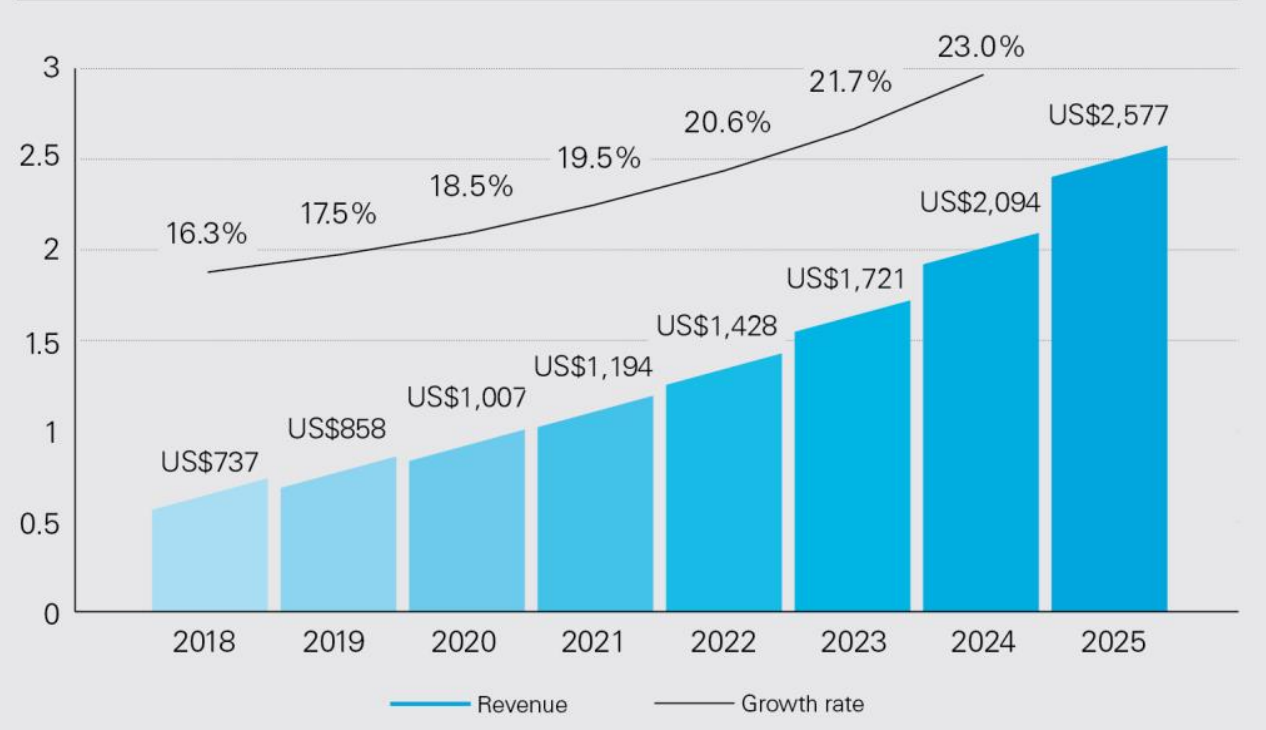

Source: Grand View Research (2018) 


\section{Fundamental Smart City Technologies}

As aforementioned, Smart Cities rely on multiple technologies to deliver sustainable socioeconomic development. People and institutions in Smart Cities can be ultra-connected. All components in Smart Cities need to work as an integrated system that can provide citizens with access to quality services in real-time (Donepudi, 2018). Such functions can only be achieved using modern technologies such as Artificial Intelligence, Internet of Things, Machine Learning and Deep Learning, among other technologies. The sections below discuss the various ways Artificial Intelligence, Internet of Things, Machine Learning and Deep Learning can be used to solve the various problems and support the development of Smart Cities.

\section{Artificial Intelligence}

Artificial Intelligence can provide numerous opportunities in the development of Smart Cities. Artificial Intelligence can help make urbanization smarter by equipping cities with advanced features. If implemented rightly, Artificial Intelligence can be life-changing (Donepudi, 2018). Below are some of the ways Artificial Intelligence can help in the implementation of smart cities.

Advanced security surveillance systems and cameras - Artificially Intelligent cameras and surveillance systems can be essential in Smart City development. They can keep an eye on the environment to provide enhanced security levels. The cameras have facial recognition capabilities that can help track unusual activities in restricted areas (Mohanty, 2016). Additionally, Artificial Intelligence cameras can monitor crowd density and track vehicles' movement throughout the day. This monitoring can help manage transport systems and congestion in cities. Most importantly, with security cameras, Smart City authorities can monitor crowds and identify possible insecurity or terrorism incidents. As a result, the authorities are able to take appropriate measures that can help thwart criminal activities in the city.

Smart Parking and Traffic Management Systems - In most cities, the number of personal and commercial vehicles is large. As a result, a huge movement of vehicles is experienced. Therefore, traffic management and getting free parking spaces is a challenge. In such situations, Artificial Intelligence can play an important role. By using road surface sensors or surveillance cameras installed in parking spaces, many cities around the world can create traffic and parking maps. The maps can help drivers identify empty parking spaces and congested roads, helping them move smoothly on roads. Additionally, with Artificial Intelligence, more riding sharing systems will be developed. Riding sharing can help decongest cities and improve citizens living in urban places with better travelling experience.

Smart waste management systems - Billions of people that live in the cities produce large volumes of waste. As a result, waste management is a big challenge in cities. Cities strive to find the best way to manage garbage in towns in order to keep the environment clean. Such challenges can be solved using Artificial Intelligence. Cities can install Artificially Intelligent sensors in waste bins. The sensors can send notifications to waste collection authorities when waste bins are nearly full. This can make waste collection more efficient and cost-effective too. The waste collection processes can be cost-effective because authorities can avoid unnecessary waste collection trips and collect waste only when bins are nearly full. 
Better city planning and governance - One of the biggest challenges in modern cities is planning and governance. However, with Artificial Intelligence, Artificial Intelligence techniques can be used to map lands in cities and generate important insights that can be used in planning. For example, cities can rely on satellite images and 2D or 3D aerial views of cities taken using artificially intelligent cameras. Most importantly, Artificial Intelligence algorithms can be used to analyze the collected satellite images and adjust planning formation based on factors such as earthquakes, floods and storms. Also, with such real-time data, management, planning and governance of cities can be done in real-time too.

Smart Healthcare - AI plays an excellent role in the healthcare industry. It can help solve various healthcare challenges faced in most cities. For example, medics can use Artificial Intelligence systems to analyze large volumes of healthcare data and provide effective healthcare services to patients. This is especially important in electronic healthcare, where medics need to provide remote healthcare to patients. With such capabilities, medics can make decisions faster and provide the appropriate healthcare services. Such efficiency can improve the lives of people, thus supporting the essence of smart cities, which is to improve the lives of citizens.

\section{Internet of Things}

In most parts of the world, the internet is used as a medium for the transmission and collection of data. However, this is changing as a large number of devices, and sensors get interconnected. The connectivity of the large number of devices that have the ability to collect data formed a basis for the development of Smart Cities (Gade et al., 2016). The use of Internet of Things applications in Smart City development will be very beneficial. The table below shows possible IoT based linkages.

Table 1: IoT Linkages

\begin{tabular}{|l|ll|}
\hline Homes & - & Health \\
& - & Entertainment \\
& - & Security \\
& - & Smart appliances \\
\hline Transport & - & Parking \\
& - & Traffic \\
& - & Emergency Services \\
\hline Society & - & Surveillance \\
& - & Environment Management \\
\hline National Uses & - & Smart Energy Management \\
& - & Smart Water Grids \\
& - & Security Management \\
\hline
\end{tabular}

Below is a discussion of some of the ways that Internet of Things will help in the development of Smart Cities.

Smart Grids - Creating smart energy sources that serve the rapidly increasing urban populations is an important step towards the construction of smart cities. A smart grid refers to computerizing power grids. This also involves the integration of digital communication technologies that support direct communication with the energy grid (Gade et al., 2016). The most important feature of Internet of Thing's smart grids is the computerization 
technology, which allows the power grid to adjust and control the millions of smart devices connected to it from a single location.

Smart Environment - Today, environmental conservation is very important. However, excellent environmental conservation cannot be achieved using the traditional methods of environmental conservation. Internet of Things has become a very useful technology in the conservation of the environment. With Internet of Things, governments are able to deploy sensors that can collect climatic data from different parts of cities. The data is collected and analyzed in real-time, helping to predict changes in climatic patterns (Gade et al., 2016). With real-time data, citizens can know more about the climate and make appropriate climate-based decisions.

Smart water management - Internet of Things is very important in the management of water systems. With IoT Systems, households connected to a smart water grid can monitor their utilization of water resources in real-time. This helps them improve water usage efficiency in Smart Cities. Water usage efficiency is important in Smart Cities because it supports the efficient usage of resources necessary in cities. Additionally, authorities can use smart IoT systems to monitor water leakages or illegal water connections. This can help combat water criminals in major cities across the world. It can also help reduce the wastage of water resources that result from water leakages.

Smart waste management - Smart waste management is important for all cities in the world. The smart management of waste can be achieved through the implementation of systems that can monitor the levels of waste in waste bins and send waste collection notifications when the bins are nearly full. This can be done by embedding sensors on trash bins so that they can report waste levels in real-time. Additionally, through Internet of Things, cities can develop IoT applications that citizens can use to know which trash bins around them are available for dumping waste. Having such applications can make their lives and the works of garbage collectors more efficient since they won't have to load bins with waste beyond their capacity. Such systems rely on both Internet of Things and Artificial Intelligence to function. They can play an essential role in the management of waste in Smart Cities.

Smart transport systems - Artificial Intelligence alone is not sufficient in the development of smart transport systems. Internet of Things can be essential due to the large number of devices connected to communication systems that report traffic flow and other transport issues. This means that large volumes of data are transmitted and analyzed to provide patterns that can be used to predict traffic movements and congestions in real-time (Gade et al., 2016). As a result, city authorities can efficiently manage traffic flow in cities.

Besides the above uses of Internet of Things, there are other ways the technology can be used in the development of Smart Cities. They include the development of smart parking applications, smart healthcare applications, and smart buildings. Generally, with billions of devices supposed to be interconnected in Smart Cities, Internet of Things will be useful. However, according to Grizhnevich (2018), some of IoT applications are not necessary for the implementation of smart systems in small cities. The table below shows IoT applications based on their relevance to small cities. 


\section{Machine Learning}

Machine Learning, like Artificial Intelligence, provides the intelligent aspect of smart city applications. First, Machine Learning can play an essential role in the healthcare sector. As mentioned earlier, good healthcare is important for the improvement of the lives of citizens living in Smart Cities (Mohapatra, 2019). For example, Machine Learning can be used in the early detection of chronic diseases. It can be used to learn the behavior of a person based on the usage of devices such as computers, smartphones, and even the use of social media sites. This can help medics tell the mental status of a person. Additionally, medics are leveraging machine learning to study diseases such as diabetes and provide useful information that can assist in providing useful and remarkable healthcare (Donepudi, 2017).

Another important application of Machine Learning in Smart Cities is that it can be used in energy management. Machine Learning can mostly be used in the prediction of solar radiation and solar output, improving the management and operation of solar power grids. Also, with Machine Learning, smart cities can monitor air quality (Mohapatra, 2019). Clean air is important for good living in cities. Cities can rely on Machine Learning applications to detect the quality of air and help them take the appropriate measures geared towards improving its quality.

Some of the Machine Learning algorithms and their uses are shown below.

\begin{tabular}{|l|l|}
\hline Algorithm & Use \\
\hline Feed Forward Neural Network & Healthcare systems \\
\hline K-means & Smart Home \\
\hline Linear Regression & Smart market analysis \\
\hline Anomaly detectors & Smart traffic \\
\hline Support vector regression & Smart weather \\
\hline
\end{tabular}

\section{Deep Learning}

Deep Learning can be used to effectively gain insights from data. It can further be used to understand data patterns and help in the classification and prediction of the data. Smart Cities can use Deep Learning in various ways. First, Deep Learning can be used in the modelling of Smart Cities. With Deep Learning, Cities can analyze data to identify developed and underdeveloped regions, then use such data to model their development plans. Additionally, Smart Cities can use Deep Learning to model intelligent infrastructure that can support billions of citizens expected to be living in cities in the future.

With Deep Learning, cities are able to gain insights about the requirements of smart cities. This can help in the efficient governance of smart cities. Most importantly, with deep learning, cities can easily understand the usage of resources such as energy and water. As a result, they can easily and efficiently manage resources. Also, Deep Learning can help in the development of useful healthcare and education applications.

\section{CONCLUSION}

Smart Cities will solve the problems associated with large populations in cities. With Smart Cities, resources will be managed efficiently. Governance and planning of cities will be efficient. Additionally, transport systems, waste management and healthcare services will be effective. However, in order to achieve such benefits, cities will need to adopt emerging 
technologies such as Artificial Intelligence, Internet of Things, Deep Learning, and Machine Learning. With such technologies, Smart Cities will be able to deliver sustainable socioeconomic development. People and institutions in Smart Cities will be ultra-connected. All components in Smart Cities will work as an integrated system that can provide citizens with access to quality services in real-time. Generally, such technologies will be the backbone for the development of Smart Cities. However, smart people and smart communities will also be necessary in order to have Smart Cities that deliver value to the people.

\section{REFERENCES}

Arroub, A., Zahi, B., Sabir, E., \& Sadik, M. (2016, October). A literature review on Smart Cities: Paradigms, opportunities and open problems. In 2016 International Conference on Wireless Networks and Mobile Communications (WINCOM) (pp. 180-186). IEEE.

Chaudhari, P. (2017). Role of Information Communication Technology (ICT) in the Development of Smart City. International Journal of Engineering Research in Computer Science and Engineering (IJERCSE). Vol 4. 271-276.

Donepudi, P. K. (2015). Crossing Point of Artificial Intelligence in Cybersecurity. American Journal of Trade and Policy, 2(3), 121-128. https://doi.org/10.18034/ajtp.v2i3.493

Donepudi, P. K. (2017). Machine Learning and Artificial Intelligence in Banking. Engineering International, 5(2), 83-86. https://doi.org/10.18034/ei.v5i2.490

Donepudi, P. K. (2018). Application of Artificial Intelligence in Automation Industry. Asian Journal of Applied Science and Engineering, 7(1), 7-20. http://doi.org/10.5281/zenodo.4146232

Gade, N. R., Gade, N. R., \& Reddy, G. U. (2016). Internet of things (IoT) for smart cities-The future technology revolution. Global Journal of Computer Science and Technology.

Grand View Research (2018). PwC and Smart Cities Market Analysis and Segment Forecats to 2025.

Grizhnevich, A. (2018). IoT for Smart Cities: Use Cases and Implementation Strategies. Science Soft. https://www.scnsoft.com/blog/iot-for-smart-city-use-cases-approaches-outcomes

Lea, Rodger. (2017). Smart Cities: An Overview of the Technology Trends Driving Smart Cities.

Mohanty, S. P., Choppali, U., \& Kougianos, E. (2016). Everything you wanted to know about smart cities: The internet of things is the backbone. IEEE Consumer Electronics Magazine, 5(3), 60-70.

Mohapatra, Badri. (2019). Machine learning applications to smart city. ACCENTS Transactions on Image Processing and Computer Vision. 5. 1-6. 10.19101/TIPCV.2018.412004.

Pozdniakova, Anna. (2016). The Concept of 'Smart City': Dimensions, Characteristics and Models.

Voda, A. I., \& Radu, L. D. (2018). Artificial intelligence and the future of smart cities. BRAIN. Broad Research in Artificial Intelligence and Neuroscience, 9(2), 110-127. 


\section{How to Cite:}

Miah, M. S., \& Amin, R. (2020). Role of Technology in the Development of Smart Cities. Engineering International, 8(1), 31-42. https://doi.org/10.18034/ei.v8i1.495 